\title{
Experimental Validation of the Quadratic Forward Model for RF Tomography
}

\author{
Vittorio Picco, Student Member, IEEE, Gianluca Gennarelli, Tadahiro Negishi, Student Member, IEEE, \\ Francesco Soldovieri, Senior Member, IEEE, and Danilo Erricolo, Senior Member, IEEE
}

\begin{abstract}
An effective way to solve the inverse scattering from dielectric objects relies on the Born approximation, which allows to linearize the problem and retrieve a qualitative reconstruction of the targets in terms of location and extent. The limits of validity of the linear model can be extended by considering a quadratic approximation of the operator relating the scattered field data to the unknown object function. The use of the quadratic operator allows on one hand to recover additional spatial variations of the object profile and on the other hand to mitigate the local minima (false solution) problem, typically affecting non-linear inversion methods. In this letter, we present an experimental validation of the quadratic inverse model for dielectric objects in free-space. The data processing confirms that the tomographic images based on the quadratic model are better resolved compared to the ones provided by the inversion of the linear Born model.
\end{abstract}

Index Terms-Born approximation, quadratic approximation, RF tomography.

\section{INTRODUCTION}

$\mathbf{R}$ ADIO Frequency (RF) tomography is a diagnostic method aiming to image targets in a scene from the electromagnetic fields scattered by them under the illumination of known incident fields [1]-[3]. As such, it is of timely interest biomedical imaging [4], ground penetrating radar imaging [5], through wall imaging [6] and urban sensing [7].

More in general, RF tomography falls within the area of electromagnetic inverse scattering [8]. Inverse scattering attempts to provide a quantitative description of the targets in terms of spatial permittivity and conductivity maps. Due to the smoothness of the radiation operator, the inverse scattering problem is ill-posed [8]. Hence, in presence of noise on data and model error, the existence and uniqueness of the solution is not ensured and a generalized solution must be searched by minimizing a suitable cost functional. Moreover, owing to the non-linear relation between data and unknowns, local deterministic minimization algorithms may be trapped into local minima leading to false solutions [9].

The mathematical formulation of RF Tomography [1]-[3] has so far relied upon the linearized model of the Born approximation. This choice offers significant advantages in terms of reduced computational complexity, stability of the solution against noise, and lack of false solutions. Additionally,

V. Picco, T. Negishi, and D. Erricolo are with the Department of Electrical and Computer Engineering, University of Illinois at Chicago, Chicago, IL 60607.

G. Gennarelli and F. Soldovieri are with the Institute for Electromagnetic Sensing of the Environment, National Research Council of Italy, Via Diocleziano 328, 80124, Napoli, Italy. the linear model allows to investigate the reconstruction performance of the adopted inversion scheme [10]. Of course, some limitations are in order. First of all, the Born approximation is valid only for weak scatterers so that only a qualitative image of the target (i.e. position and approximate shape) can be attained in most practical cases. Secondly, the Born model neglects the mutual coupling among targets and the interactions with surrounding environment thus introducing artifacts or ghost targets [11]. A third aspect is the spatial filtering introduced by the linear scattering operator, which implies a limitation on the achievable resolution [12].

Improved approximations such as the extended Born approximation [13], the quasi-linear approximation [14], the diagonal tensor approximation [15] were proposed to overcome the limitations of the Born approximation. Several non-linear inversion methods were also presented (e.g. see [16]-[21]), but their applicability in realistic scenarios still remains an open subject. An assessment of different inversion approaches based on the Fresnel database was reported in [22].

In this letter, we consider a second order Born approximation to extend the class of retrievable spatial variations of the object function, which leads to a quadratic forward model [23]-[25]. In particular, the imaging problem is cast as the minimization of a quartic functional. The quadratic model has the benefit that local minima can be avoided by suitably increasing the ratio between the number of data and unknowns [9]. Note that works [23]-[25] were mainly conceived as theoretical studies with source and receivers located in the far-zone with respect to the investigation domain. This letter extends the previous analysis to near-zone measurement configurations providing experimental evidence of the imaging improvement offered by the quadratic model in the case of realistic targets. This analysis has not been reported to date and complements preliminary numerical authors' studies [26].

\section{Problem statement}

The problem is tackled in a $2 \mathrm{D}$ space as shown in Fig. 1 and the time dependence $e^{j 2 \pi f t}$ is assumed and suppressed. The objects to be imaged reside in the investigation domain $D$ and are assumed invariant along the $z$-axis. The transmitting and receiving antennas operate at fixed frequency $f$ and are modeled as filamentary electric currents infinitely long and spatially invariant along the $z$-axis. The position of transmitters and receivers, here denoted by $\mathbf{r}^{\mathrm{s}}$ and $\mathbf{r}^{\mathrm{r}}$, are moved over the measurement domain $\Gamma$ so that a multiview/multistatic single frequency configuration is achieved. The background medium 


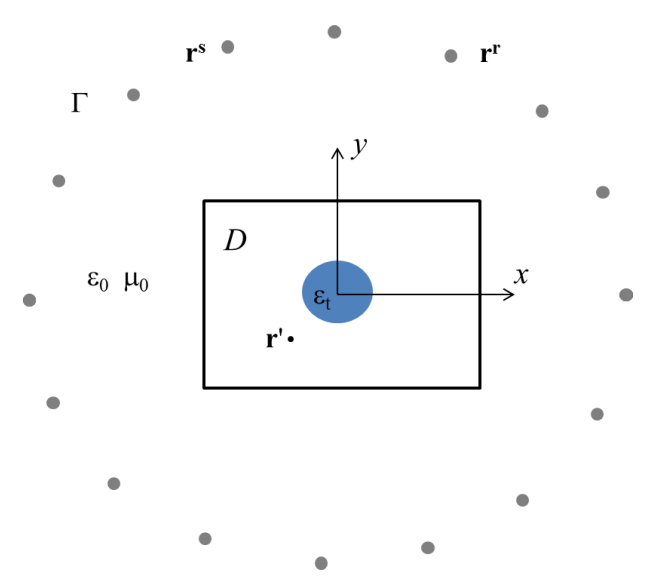

Fig. 1. The inverse scattering problem. The rectangular area $D$ represents the investigation domain. Gray circles denote the transmitting/receiving antennas.

is assumed to be free-space with dielectric permittivity $\varepsilon_{0}$ and magnetic permeability $\mu_{0}$. The targets are made of a lossless non-magnetic $\left(\mu=\mu_{0}\right)$ dielectric material with real permittivity $\varepsilon_{t}$. They are described by the contrast function

$$
\chi\left(\mathbf{r}^{\prime}\right)=\frac{\varepsilon\left(\mathbf{r}^{\prime}\right)-\varepsilon_{0}}{\varepsilon_{0}},
$$

where $\mathbf{r}^{\prime}$ and $\varepsilon\left(\mathbf{r}^{\prime}\right)$ represent a generic point and the permittivity function in $D$, respectively.

The scattering phenomenon can be described by two scalar integral equations [27]. The first one is the external equation which relates the scattered field $E^{\mathrm{s}}$ (data) to the contrast function $\chi$ (unknown)

$$
E^{\mathrm{s}}\left(\mathbf{r}^{\mathrm{r}}, \mathbf{r}^{\mathrm{s}}\right)=k_{0}^{2} \iint_{D} \chi\left(\mathbf{r}^{\prime}\right) E^{\mathrm{t}}\left(\mathbf{r}^{\prime}, \mathbf{r}^{\mathrm{s}}\right) g_{e}\left(\mathbf{r}^{\mathrm{r}}, \mathbf{r}^{\prime}\right) d \mathbf{r}^{\prime},
$$

where $k_{0}=2 \pi f / c_{0}$ is the propagation constant in free-space ( $c_{0}$ is the wavespeed), $E^{\mathrm{t}}\left(\mathbf{r}^{\prime}, \mathbf{r}^{\mathrm{s}}\right)$ is the total field in $D$ and

$$
g_{e}\left(\mathbf{r}^{\mathrm{r}}, \mathbf{r}^{\prime}\right)=-\frac{j}{4} H_{0}^{(2)}\left(\mathbf{r}^{\mathrm{r}}, \mathbf{r}^{\prime}\right)
$$

is the external Green's function. $H_{0}^{(2)}(\cdot)$ is the Hankel function of second kind of zero order.

The total field $E^{\mathrm{t}}$ in $D$ is related to $\chi$ by the internal equation

$E^{\mathrm{t}}\left(\mathbf{r}^{\prime}, \mathbf{r}^{\mathbf{s}}\right)=E^{\mathrm{i}}\left(\mathbf{r}^{\prime}, \mathbf{r}^{\mathrm{s}}\right)+k_{0}^{2} \iint_{D} \chi\left(\mathbf{r}^{\prime \prime}\right) E^{\mathrm{t}}\left(\mathbf{r}^{\prime \prime}, \mathbf{r}^{\mathrm{s}}\right) g_{i}\left(\mathbf{r}^{\prime}, \mathbf{r}^{\prime \prime}\right) d \mathbf{r}^{\prime \prime}$,

where $E^{\mathrm{i}}$ is the incident field (i.e. the field in absence of targets) and $g_{i}\left(\mathbf{r}^{\prime}, \mathbf{r}^{\prime \prime}\right)$ is the internal Green's function [28]. According to eq. (4), the total field is the superposition of two contributions. The first one is the incident field whereas the second term, defined as an integral over $D$, accounts for mutual interactions between different scatterers or between the same parts of a scatterer [24], [25], [27].

Equations (2) and (4) can be rewritten in operator form as

$$
\begin{aligned}
& E^{\mathrm{s}}=\mathbf{A}_{\mathrm{e}}\left[\chi E^{\mathrm{t}}\right] \\
& E^{\mathrm{t}}=E^{\mathrm{i}}+\mathbf{A}_{\mathrm{i}}\left[\chi E^{\mathrm{t}}\right]
\end{aligned}
$$

where $\mathbf{A}_{\mathrm{e}}$ and $\mathbf{A}_{\mathrm{i}}$ represent the external and internal radiation operators. The above expressions can be manipulated in order to obtain the functional relation

$$
E^{\mathrm{s}}=\mathbf{A}_{\mathrm{e}}\left[\chi\left(\mathbf{I}-\mathbf{A}_{\mathrm{i}} \chi\right)^{-1} E^{\mathrm{i}}\right] .
$$

where $\mathbf{I}$ is the identity operator.

According to eq. (7), a nonlinear relationship is established between the unknown contrast function and the scattered field data. Nonlinear inversion procedures allow in principle a quantitative reconstruction of the contrast function [16][19]. However, deterministic minimization algorithms may be trapped into local minima yielding false solutions [9], [18], [24]. On the other hand, the application of global optimization schemes is often not viable due to the high computation cost when a great number of unknowns must be searched for.

\section{INVERSION APPROACHES: LINEAR AND QUADRATIC MODEL}

Approximate linearized models can be exploited when qualitative reconstructions are of concern. The most usual one is based on Born approximation [27]. In particular, under the assumption that $\left\|\mathbf{A}_{\mathrm{i}} \chi\right\| \ll 1$, it is possible to expand around the origin the nonlinear relation (7) as

$$
\left(\mathbf{I}-\mathbf{A}_{\mathrm{i}} \chi\right)^{-1}=\mathbf{I}+\mathbf{A}_{\mathrm{i}} \chi+\left(\mathbf{A}_{\mathrm{i}} \chi\right)^{2}+\ldots+\left(\mathbf{A}_{\mathrm{i}} \chi\right)^{n},
$$

The Born approximation consists in dropping all but the first term in the summation

$$
E^{\mathrm{s}} \approx \mathbf{A}_{\mathrm{e}}\left[\chi E^{\mathrm{i}}\right]=\mathbf{A}[\chi]
$$

i.e. approximating the total field in $D$ as the incident field. Eq. (9) is linear with respect to $\chi$ and different schemes can be used to invert it [10]. In addition, even if the Born model strictly holds for weak scatterers, it can be relaxed and applied also to the shape reconstruction of non weakly scattering targets [1], [29].

A possibility to improve the accuracy of the model is to retain the second term of the series in (8). This leads to a quadratic model with respect to the unknown $\chi[23]-[25]$ :

$$
E^{\mathrm{s}} \approx \mathbf{A}_{\mathrm{e}}\left[\chi E^{\mathrm{i}}\right]+\mathbf{A}_{\mathrm{e}}\left[\chi \mathbf{A}_{\mathrm{i}}\left[\chi E^{\mathrm{i}}\right]\right]
$$

The introduction of the quadratic term has the benefit of providing a better approximation of the scattering phenomenon [23]-[25]. Moreover, it has been shown to enhance the spatial resolution because additional spatial harmonics beat each other and their folding maps at base band (i.e. the range of the linear operator A). The interested reader can refer to [24], [25] for a more detailed explanation of this phenomenon. However, the resolution improvement comes at the cost of a major computation complexity and the introduction of a non-linearity that can produce local minima in the inversion stage. According to [9], [18], the local minima problem can be overcome if the ratio between the number of data and number of unknowns is sufficiently large. Turning to the numerical implementation of the inversion methods, the contrast function is here represented in terms of a finite Fourier series [25]: 


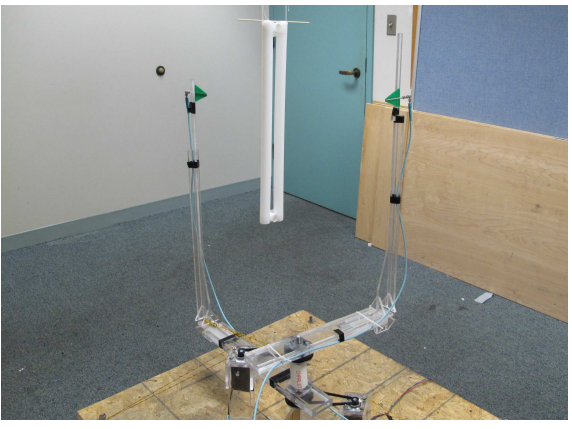

Fig. 2. Photo of the experimental set-up.

$$
\chi(x, y)=\sum_{m=-M}^{M} \sum_{n=-N}^{N} c_{m n} e^{j 2 \pi m x / L_{x}} e^{j 2 \pi n y / L_{y}},
$$

where

$$
c_{m n}=\frac{1}{L_{x} L_{y}} \iint \chi(x, y) e^{-j 2 \pi m x / L_{x}} e^{-j 2 \pi n y / L_{y}} d x d y .
$$

In eqns. (11) and (12), $x$ and $y$ are the coordinates of a point in the investigation domain, $L_{x}$ and $L_{y}$ are the sizes of $D, m$ and $n$ are the indexes of the Fourier coefficients $c_{m n}$, and $2 M+1$ and $2 N+1$ are the total number of coefficients to be searched for along $x$ and $y$, respectively.

The inversion task is formulated as an optimization problem where a cost functional has to be globally minimized over the coefficients $c_{m n}$. The cost functionals for both linear and the quadratic cases are so defined:

$$
\begin{aligned}
& \Psi^{\text {Born }}(\mathbf{c})=\left\|E^{\mathrm{s}}-\mathbf{A}_{\mathrm{e}}\left[\chi E^{\mathrm{i}}\right]\right\|^{2}, \\
& \Psi^{\text {quad }}(\mathbf{c})=\left\|E^{\mathrm{s}}-\mathbf{A}_{\mathrm{e}}\left[\chi E^{\mathrm{i}}\right]-\mathbf{A}_{\mathrm{e}}\left[\chi \mathbf{A}_{\mathrm{i}}\left[\chi E^{\mathrm{i}}\right]\right]\right\|^{2} .
\end{aligned}
$$

where $\mathbf{c}=\left\{c_{m n}\right\}_{m=-M, \ldots, M}^{n=-N, \ldots, N}$ is the vector of unknowns.

A Levenberg-Marquardt algorithm is adopted in this study to minimize the functionals in eqns. (13) and (14). The algorithm is implemented by the MATLAB built-in function lsqnonlin, which solves non-linear least-squares problems. The maximum number of iterations is set at 400 (default value) and the termination tolerance on the functional value is equal to $1 \mathrm{e}-6$. The degree of the regularization is imposed by searching for a finite number of Fourier harmonics, which implies the smoothness of the solution. Once the vector $\mathbf{c}$ is calculated, the contrast function $\chi$ is recovered via eq. (11). The spatial map defined by the magnitude of $\chi$ is herein referred to as tomographic image.

\section{EXPERIMENTAL SET-UP}

The experiments were conducted in the Andrew Electromagnetic Laboratory at the University of Illinois at Chicago. The antenna positioning system is depicted in Fig. 2 and consists of two stepper motors that move two antennas around concentric circles, guaranteeing a positioning precision smaller than $1^{\circ}$. A computer coordinates the movement of the motors and the measurement of the electric field at each antenna position. For each transmitter position, all receiver positions

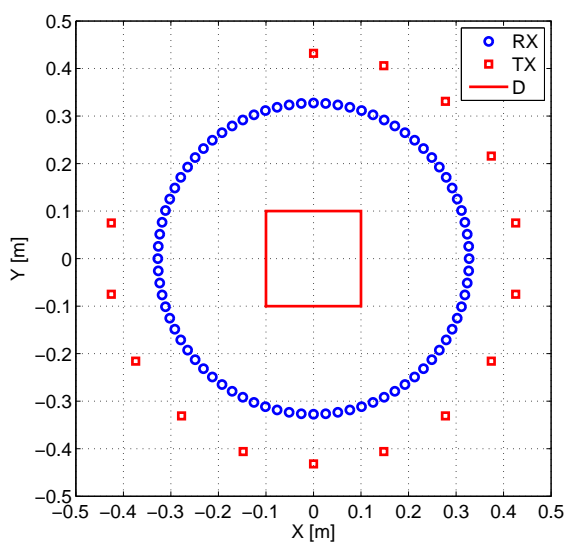

Fig. 3. Sketch of the measurement configuration. Red squares denote the transmitters while blue circles are the receivers.

are sampled so that a multiview/multistatic configuration is obtained. The transmitting and receiving antennas are logperiodic (model Ramsey LPY26) and operate at fixed frequency of $5 \mathrm{GHz}$, which corresponds to a propagating wavelength $\lambda$ equal to $6 \mathrm{~cm}$. The data are collected by using a vector network analyzer (VNA, model HP 8753ES).

As shown in Fig. 3, the transmitting antenna moves around the outer circle of radius $43.2 \mathrm{~cm}(7.2 \lambda)$, illuminating the target from 15 positions uniformly spaced along an arc spanning $280^{\circ}$. The receiving antenna moves around the inner circle of radius $32.8 \mathrm{~cm}(5.5 \lambda)$, sampling the field at 80 positions uniformly spaced along the circle.

As well-known, the VNA measures the scattering parameter $S_{12}$ accounting for transmission between the transmitting and receiving antennas. This parameter is recast in terms of scattered electric field according to the following two steps: background subtraction and calibration.

Background subtraction works as follows. For every measurement, two sets of data are collected: in the first set no object is present in the area under investigation, thus obtaining $S_{12}^{\mathrm{i}}$; in the second set the object to reconstruct is placed in the area under investigation and values of $S_{12}^{\mathrm{t}}$ are collected at the same positions used in the first set. The two datasets are then subtracted obtaining $S_{12}^{\mathrm{s}}$, which is a measure of the scattered electric field. Note that the stepper motors allow high precision in the positioning of the antennas to guarantee repeatability of the measurements.

In order to obtain the scattered electric field $E^{s}$ from $S_{12}^{\mathrm{s}}$, a calibration stage is performed. First, for a given transmitter position $\mathbf{r}^{\mathrm{s}}$ and receiver position $\mathbf{r}^{\mathrm{r}}$, the incident electric field is calculated as

$$
E^{\mathrm{i}}\left(\mathbf{r}^{\mathrm{r}}\right)=-\frac{k_{0}^{2}}{4 \omega \varepsilon_{0}} H_{0}^{(2)}\left(k_{0}\left|\mathbf{r}^{\mathrm{r}}-\mathbf{r}^{\mathrm{s}}\right|\right)
$$

Then, the scattered electric field $E^{s}$ at each receiver position is obtained as

$$
E^{\mathrm{s}}\left(\mathbf{r}^{\mathrm{r}}\right)=\frac{E^{\mathrm{i}}\left(\mathbf{r}^{\mathrm{r}}\right)}{S_{12}^{\mathrm{i}}} S_{12}^{\mathrm{s}}
$$

\footnotetext{
${ }^{1}$ The mechanical set-up does not allow for a full $360^{\circ}$ rotation.
} 

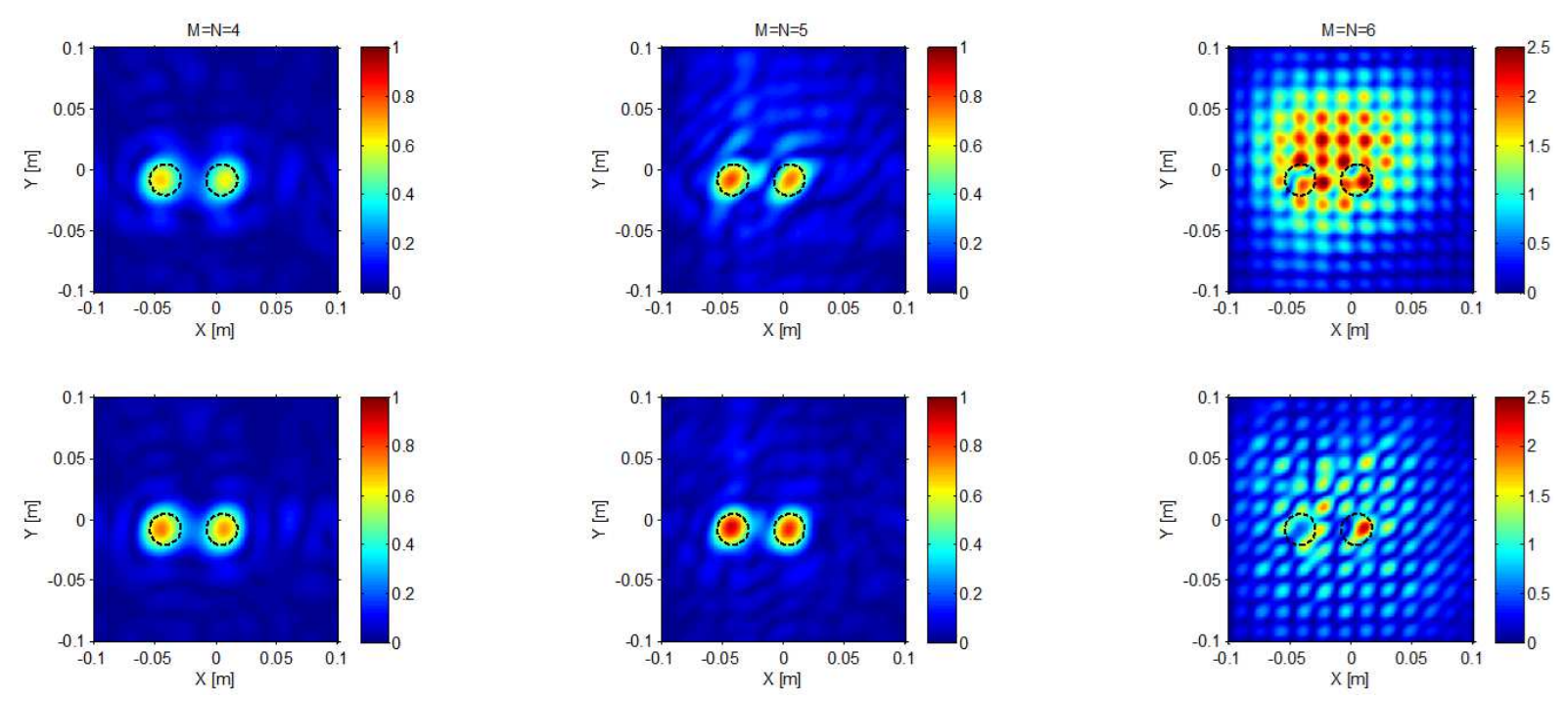

Fig. 4. Tomographic reconstructions of the two HDPE cylinders at $5 \mathrm{GHz}$ versus $M$ and $N$. Linear model (upper panels). Quadratic model (lower panels).

This procedure is reliable provided that $S_{12}^{\mathrm{i}}$ can be measured with reasonable accuracy. In practice, not all antenna locations are equally good for this purpose, because some are disturbed by the presence of the antenna positioner itself. The most reliable values of $S_{12}^{\mathrm{i}}$ are measured when transmitter and receiver antennas are facing each other. Therefore, for each transmitter position, the values of $E^{\mathrm{i}}\left(\mathbf{r}^{\mathrm{r}}\right)$ and $S_{12}^{\mathrm{i}}$ corresponding to the opposite receiver are used in eq. (16).

The investigation domain $D$ is a square of side equal to $20 \mathrm{~cm}(3.3 \lambda)$, which is partitioned into square pixels of side approximately equal to $1.2 \mathrm{~cm}(\lambda / 5)$.

\section{RECONSTRUCtion RESUlts}

Two experimental tests are described in this Section. The first one pertains to two high-density polyethylene cylinders (HDPE, $\left.\varepsilon_{t} \approx 2.26 \varepsilon_{0}\right)$ of diameter $2.54 \mathrm{~cm}(0.42 \lambda)$, whose outer edges are spaced by $2 \mathrm{~cm}(0.33 \lambda)$. The inversions have been performed by searching for different values of $M$ and $N$ ( $M=N=4, M=N=5$, and $M=N=6$ ) to analyze how the number of unknowns impacts on the achieved solution.

The images in Fig. 4 confirm that, when $M=N=4$ and $M=N=5$, both Born and quadratic models lead to satisfactory results in terms of position and shape reconstruction capabilities. In particular, the focusing performance of linear and quadratic models look similar for $M=N=4$. However, when $M=N=5$, the inversion performed according to the quadratic model is clearly superior to the one obtained with the Born approximation. Indeed, it is easily recognized that the contours of the cylinders are more clearly defined and the image has less artifacts overall. This is because when searching for a higher number of coefficients, spatial harmonics outside the range of the linear operator can be imaged by the quadratic operator [25]. When $M=N=6$, both models produce an unstable solution due to the ill-posedness of the inverse scattering problem. It must be also noticed that, when $M=N=4$ and $M=N=5$, the value of the reconstructed contrast in the quadratic case is slightly larger than in linear case, i.e. the solution of quadratic approach is closer to the true contrast $(\chi=1.26)$. However, both solutions under-estimate the true contrast because the considered HDPE cylinders are beyond the limit of validity of both Born and quadratic models $\left(\left\|\mathbf{A}_{\mathrm{i}} \chi\right\|=15.1\right)$. This behavior has been confirmed by numerical simulations reproducing the experiment, whose results are omitted for space limitation. The similarity between synthetic and real data inversion has also confirmed that the data are mainly affected by model error. Indeed, the SNR of the measurement system has been estimated around $53 \mathrm{~dB}$ for the scattered field.

The second test-case refers to an L-shaped object made of plexiglass $\left(\varepsilon_{t} \approx 2.6 \varepsilon_{0}\right)$. The object is $6.9 \mathrm{~cm}(1.15 \lambda)$ and $5.6 \mathrm{~cm}(0.93 \lambda)$ long along $x$ and $y$ respectively, and the thickness of each arm is about $0.5 \mathrm{~cm}(0.08 \lambda)$. The most accurate solution is attained when $M=N=4$ (see Figs. 5 and 6). In spite of the small thickness of the L arms, which is lower than system resolution, the reconstructions of Figs. 5 and 6 confirm the superior focusing of the quadratic model with respect to the linear one. The model error is not negligible also in this case $\left(\left\|\mathbf{A}_{\mathrm{i}} \chi\right\|=9.9\right)$, thus the retrieved contrast does not allow to estimate the target permittivity.

The resolution improvement provided by the quadratic model is quantified in terms of the normalized sum of squared intensity:

$$
C=\frac{\sum_{q=1}^{Q}\left|\chi\left(x_{q}, y_{q}\right)\right|^{4}}{\left(\sum_{q=1}^{Q}\left|\chi\left(x_{q}, y_{q}\right)\right|\right)^{4}}
$$

where $\left(x_{q}, y_{q}\right)$ are the coordinates of pixel $q$ in the image, and $Q$ is the total number of pixels. Note that a greater value of $C$ correspond to a higher image sharpness. The $C^{\text {quad }} / C^{\text {Born }}$ ratio summarized in Tab. I confirm that the tomographic images obtained with the quadratic model always have higher sharpness than those achieved inverting the linear model, save for the the L-shaped target when $M=N=5$ because the quadratic solution (not shown) is approaching instability. 


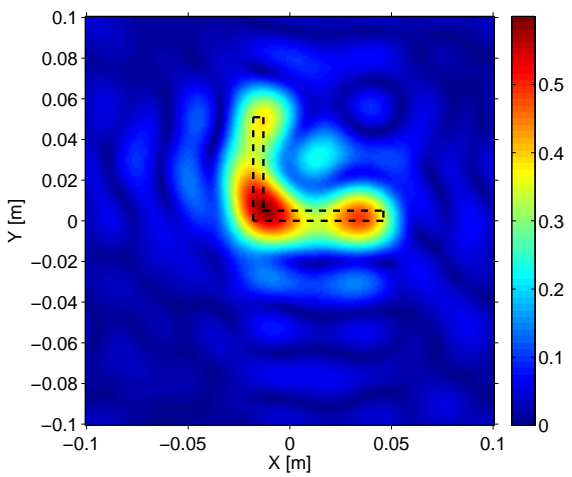

Fig. 5. Tomographic reconstructions of the L-shaped plexiglass object at 5 GHz. Linear model using $M=N=4$.

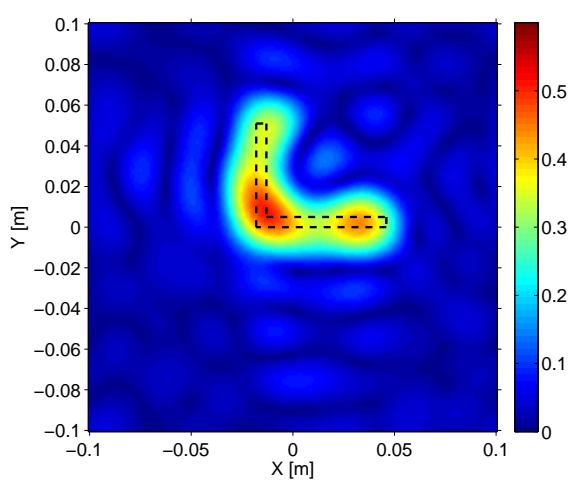

Fig. 6. Tomographic reconstructions of the L-shaped plexiglass object at 5 GHz. Quadratic model using $M=N=4$.

\section{CONCLUSION}

We have proposed an experimental validation of the quadratic model for RF Tomography. The object function has been represented as a finite summation of spatial Fourier harmonics, and the forward model has been inverted by minimizing a cost functional using a Levenberg-Marquardt algorithm. For comparison, the same inversion procedure has been applied to the linear Born model. The quadratic model generally provides superior performance in terms of artifact mitigation and spatial resolution. However, its validity is limited by the constraint $\left\|\mathbf{A}_{\mathrm{i}} \chi\right\| \ll 1$, which restricts the class of targets that can be reconstructed in a quantitative manner.

\section{ACKNOWLEDGEMENT}

This work was partially supported by the AFRL through grant FA9550-12-1-0174.

\section{REFERENCES}

[1] L. Lo Monte, D. Erricolo, F. Soldovieri, and M. C. Wicks, "Radio frequency tomography for tunnel detection," IEEE Trans. Geosci. Remote Sensing, vol. 48, no. 3, pp. 1128-1137, Mar 2010.

[2] — , "Rf tomography for below-ground imaging of extended areas and close-in sensing," IEEE Geosci. Rem. Sens. Lett., vol. 7, no. 3, pp. 496500, Jul 2010.

[3] L. Lo Monte, F. Soldovieri, D. Erricolo, and M. C. Wicks, "Imaging below irregular terrain using rf tomography," IEEE Trans. Geosci. Remote Sensing, vol. 50, no. 9, pp. 3364-3373, Sep 2012.

[4] N. K. Nikolova, "Microwave imaging for breast cancer," IEEE Micr. Mag., vol. 12, no. 7, pp. 78-94, 2011.
TABLE I

NORMALIZED SUM OF SQUARED INTENSITY RATIO

\begin{tabular}{|c|c|}
\hline Test case & $C^{\text {quad }} / C^{\text {Born }}$ \\
\hline Two HDPE cylinders $(\mathrm{M}=\mathrm{N}=4)$ & 1.57 \\
\hline Two HDPE cylinders $(\mathrm{M}=\mathrm{N}=5)$ & 4.55 \\
\hline L-shaped plexiglass object $(\mathrm{M}=\mathrm{N}=4)$ & 1.22 \\
\hline L-shaped plexiglass object $(\mathrm{M}=\mathrm{N}=5)$ & 0.45 \\
\hline
\end{tabular}

[5] R. Persico, Introduction to ground penetrating radar: inverse scattering and data processing. John Wiley \& Sons, 2014.

[6] M. G. Amin, Through-the-wall radar imaging. CRC press, 2011.

[7] G. Gennarelli, I. Catapano, and F. Soldovieri, "Rf/microwave imaging of sparse targets in urban areas," IEEE Ant. Wir. Propag. Lett., vol. 12, pp. 643-646, 2013.

[8] D. Colton and R. Kress, Inverse acoustic and electromagnetic scattering theory. Springer, 2012, vol. 93.

[9] T. Isernia, F. Soldovieri, G. Leone, and R. Pierri, "On the local minima in phase reconstruction algorithms," Rad. Sci., vol. 31, no. 6, pp. 1887$1899,1996$.

[10] M. Bertero and P. Boccacci, Introduction to inverse problems in imaging. CRC press, 1998.

[11] G. Gennarelli and F. Soldovieri, "Multipath ghosts in radar imaging: physical insight and mitigation strategies," IEEE Journ. Sel. Top. Ear. Obs. Rem. Sens., p. (in print).

[12] E. Wolf, "Three-dimensional structure determination of semi-transparent objects from holographic data," Opt. Comm., vol. 1, no. 4, pp. 153-156, 1969.

[13] T. M. Habashy, R. W. Groom, and B. R. Spies, "Beyond the born and rytov approximations: A nonlinear approach to electromagnetic scattering," Journal of Geophysical Research: Solid Earth (1978-2012), vol. 98, no. B2, pp. 1759-1775, 1993.

[14] M. S. Zhdanov and S. Fang, "Quasi-linear approximation in 3-d electromagnetic modeling," Geophysics, vol. 61, no. 3, pp. 646-665, 1996.

[15] L.-P. Song and Q. H. Liu, "Fast three-dimensional electromagnetic nonlinear inversion in layered media with a novel scattering approximation," Inv. Probl., vol. 20, no. 6, p. S171, 2004.

[16] W. Chew and Y. Wang, "Reconstruction of two-dimensional permittivity distribution using the distorted born iterative method," IEEE Trans. Med. Imag., vol. 9, no. 2, pp. 218-225, 1990.

[17] N. Joachimowicz, C. Pichot, and J.-P. Hugonin, "Inverse scattering: An iterative numerical method for electromagnetic imaging," IEEE Trans. Ant. Propag., vol. 39, no. 12, pp. 1742-1753, 1991.

[18] T. Isernia, V. Pascazio, and R. Pierri, "A nonlinear estimation method in tomographic imaging," IEEE Trans. Geosci. Rem. Sens., vol. 35, no. 4, pp. 910-923, 1997.

[19] P. M. Van Den Berg and R. E. Kleinman, "A contrast source inversion method," Inverse problems, vol. 13, no. 6, p. 1607, 1997.

[20] A. Abubakar, T. M. Habashy, P. M. van den Berg, and D. Gisolf, "The diagonalized contrast source approach: an inversion method beyond the born approximation," Inv. Probl., vol. 21, no. 2, p. 685, 2005.

[21] X. Chen, "Subspace-based optimization method for solving inversescattering problems," IEEE Trans. Geosci. Rem. Sens., vol. 48, no. 1, pp. 42-49, Jan 2010.

[22] K. Belkebir and M. Saillard, "Guest editors' introduction: Testing inversion algorithms against experimental data: inhomogeneous targets," Inv. Probl., vol. 21, p. 1, 2005.

[23] G. Leone, A. Brancaccio, and R. Pierri, "Linear and quadratic inverse scattering for angularly varying circular cylinders," JOSA A, vol. 16, no. 12 , pp. 2887-2895, 1999.

[24] R. Pierri and G. Leone, "Inverse scattering of dielectric cylinders by a second-order born approximation," IEEE Trans. Geosci. Rem. Sens., vol. 37, no. 1, pp. 374-382, 1999.

[25] R. Pierri, F. Soldovieri, A. Liseno, and F. De Blasio, "Dielectric profiles reconstruction via the quadratic approach in 2-d geometry from multifrequency and multifrequency/multiview data," IEEE Trans. Geosci. Rem. Sens., vol. 40, no. 12, pp. 2709-2718, 2002.

[26] V. Picco, G. Gennarelli, T. Negishi, D. Erricolo, and F. Soldovieri, "RF Tomography Under the Quadratic Approximation," in IEEE Antennas and Propagation Society International Symposium/USNC-URSI National Radio Science Meeting, Memphis, TN, USA, July 6-11 2014.

[27] W. C. Chew, Waves and fields in inhomogeneous media. IEEE press New York, 1995, vol. 522. 
[28] J. Richmond, "Scattering by a dielectric cylinder of arbitrary cross section shape," IEEE Trans. Ant. Propag., vol. 13, no. 3, pp. 334-341, 1965.

[29] R. Solimene, I. Catapano, G. Gennarelli, A. Cuccaro, A. Dell'Aversano, and F. Soldovieri, "Sar imaging algorithms and some unconventional applications," IEEE Sign. Proc. Mag., vol. 31, no. 4, pp. 90-98, 2014. 